\title{
Pulsed high power fiber laser systems
}

\author{
A. Malinowski, J.H.V. Price, F. He, P.Dupriez, H.D.Foreman ${ }^{\dagger}$, A.C.Tropper ${ }^{\dagger}$, J. Nilsson* and \\ D.J. Richardson* \\ Optoelectronics Research Centre (ORC), University of Southampton, Southampton SO17 1BJ, \\ United Kingdom. \\ ${ }^{\dagger}$ Department of Physics, University of Southampton, Southampton SO17 1BJ, United Kingdom. \\ *also with SPI, 3 Wellington Park, Tollbar Way, Hedge End, Southampton, SO30 $2 Q U$. \\ Tel: +442380 594523,Fax:+442380593142,Email:anm@orc.soton.ac.uk
}

Summary: The Master-Oscillator Power-Amplifier approach, cladding pumping technology and large mode area fibers provide a route to compact and efficient pulsed fiber laser systems with high beam quality and high output powers.

There is an increasing demand for high-power pulsed laser systems for industrial and scientific applications. Diode pumped rare-earth doped fiber laser technology is attractive for high average power systems because of high conversion efficiency (70-80\% for Yb-doped fiber) and because of its good thermal management qualities. Cladding pumping allows very high pump powers to be coupled to the gain medium. Output powers of more than $2 \mathrm{~kW}$ in a single transverse mode have now been achieved in c.w. fiber lasers. Furthermore, the broad gain bandwidths of Er and $\mathrm{Yb}$ doped fibers can support $\sim 100 \mathrm{fs}$ pulses. However, the same qualities which make fibers attractive for high average power operation (tightly confined mode, long gain length) present challenges for pulsed operation. Peak power is capped by Raman scattering and the pulses can be distorted by SPM due to the relatively strong effective nonlinearity, and for ultrashort pulses the fiber dispersion must be managed to maintain short pulse duration.

Large Mode Area (LMA) fibers[1], which allow (near) single mode operation in multimode fiber, are crucial for obtaining the highest possible peak powers and pulse energies in fiber systems. Large mode area core designs facilitate power handling (e.g. facet damage threshold), reduce nonlinearity (increasing thresholds for SPM and Raman), have high energy storage, facilitate efficient pump absorption and reduce device lengths

The MOPA (Master Oscillator- Power Amplifier) is a powerful and versatile approach for fiber laser systems. The MOPA configuration allows a generic high power amplifier to be combined with a wide variety of different pulse sources. It is relatively easy to control the characteristics of pulses at low power, and the efficiency of the Master Oscillator is not an issue. A wide variety of sources are available (both fiber and non-fiber), with a huge range of pulse durations and repetition rates, which are suitable for seeding a fiber amplifier. Fiber amplifiers are simple, robust and have a unique combination of high gain, high power, and high efficiency. Some examples of our work on high power fiber MOPAs are described below.

Fiber pigtailed laser diodes are very simple and convenient sources of nanosecond pulses for a fiber amplifier. They can be directly electrically modulated to produce nanosecond pulses, or gain switched to produce high repetition rate picosecond pulses (see below). The combination of telecommunication-grade, low-power laser diodes and fiber-based power-amplifier systems constitutes an attractive approach to the development of versatile, robust, and compact high power pulsed sources.

Nanosecond pulsed fiber MOPAs with pulse energies in the region of $1 \mathrm{~mJ}$ are promising sources for materials processing applications. In the past we have used Q-switched fiber lasers as nanosecond sources for fiber amplifier[2], but with a laser diode control over pulse duration is much greater. To achieve high energies and peak powers in this scheme requires gains of order 40-50 dB. With this level of gain significant pulse reshaping can take place in the amplifier chain. We experimentally observed and successfully modeled gain-shaping of pulses in a fiber amplifier chain with $>40 \mathrm{~dB}$ total gain at $10-200 \mathrm{kHz}$ repetition rate and $20-200 \mathrm{~ns}$ pulse durations. We obtained pulse energies of up to $0.6 \mathrm{~mJ}$. The initial 100ns square pulse was reduced to an effective pulse duration of $\sim 20 \mathrm{~ns}$ due to gain reshaping, with peak power greater than $30 \mathrm{~kW}[3]$.

Whilst material processing often relies on high energy per pulse, other applications such as nonlinear frequency conversion for laser projection require good beam quality, narrow spectral linewidth, and high peak power. Free-space communication requires sources generating high data rate, high peak power and excellent beam quality. A high repetition rate picosecond source is attractive for obtaining clean pulses with good extinction ratio even at high data rates. Furthermore the power damage threshold is higher for picosecond pulses than for nanosecond pulses. Thus, a high repetition rate picosecond source allows for both high peak power and high average power. Gain switching of laser diodes has proved to be a convenient and practical method to generate high repetition rate 
picosecond pulses. Gain-switched diode lasers were primarily developed for telecommunications applications at limited pulse powers. However, recently they have also been used as seed lasers for high-power cladding-pumped erbium:ytterbium doped fiber master oscillator - power amplifier (MOPA) sources emitting at $1.55 \mu \mathrm{m}$ [4]. Recent developments in laser diodes emitting around $1-1.1$ $\mu \mathrm{m}$ has now permitted an extension of this approach to ytterbium-doped fiber MOPA systems. We developed a MOPA source consisting of a gain-switched laser diode emitting at $1060 \mathrm{~nm}$ and a Ybdoped fiber amplifier chain. The pulses from the diode were compressed to 20ps with a fiber grating and amplified in a 4-stage $\mathrm{Yb}$ fiber amplifier chain to $321 \mathrm{~W}$ of average output power[5]. Similar systems were recently used to pump a Raman fiber amplifier operating at $1178 \mathrm{~nm}$, which was frequency doubled to produce output at $589 \mathrm{~nm}[6]$, and to creat an $80 \mathrm{~W}$ green source by frequency doubling the amplifier output[7].

Thanks to their stability, compactness and capability to produce very clean short pulses in a diffraction limited output beam, passively mode-locked VECSELs (Vertical External Cavity Surface Emitting Lasers) are well suited to power scaling through amplification in fibers. As a source, we used a VECSEL with a center wavelength of $1054.6 \mathrm{~nm}$ which emitted 4.6-ps pulses with a repetition frequency of $910 \mathrm{MHz}$ and an average power of $8 \mathrm{~mW}$. The pulses were amplified in a 3-stage $\mathrm{Yb}$ amplifier chain culminating in a 12-m-long Yb-doped double-clad fiber, with core and cladding diameters of $25 \mu \mathrm{m}$ and $400 \mu \mathrm{m}$ respectively. The maximum output power was $255 \mathrm{~W}[8]$. Thanks to the low core NA $(<0.05)$ and proper fundamental mode excitation the output beam was diffraction limited with $\mathrm{M}^{2}<1.1$. It was estimated that the output pulse train accounted for about $95 \%$ of the output power, corresponding to a pulse energy of $196 \mathrm{~nJ}$ and a peak power of $38 \mathrm{~kW}$. The spectrum of the pulse was broadened due to SPM during amplification. A fraction of the pulse output power was coupled into a free-space bulk grating compressor, where the separation was adjusted to minimize the width of the compressed pulses. The width of the compressed pulses at $200 \mathrm{~W}$ was estimated $\sim 400 \mathrm{fs}$. Further enhancement of this scheme should allow us to operate in the parabolic amplification regime, yielding clean $\sim 100 \mathrm{fs}$ pulses. Much higher repetition rates are also possible.

We have also worked with femtosecond modelocked fiber lasers as seeds for fiber amplifiers[9]. The three-level nature of the Yb-system causes the wavelength dependant gain profile to vary significantly depending on the interplay of pump and signal power, wavelength, amplifier length, and pump configuration, making the design and optimisation of an amplifier system for a given application a complex problem. Optimising the overall gain bandwidth is of critical importance for high power ultra-short pulse chirp-pulse-amplifier (CPA) [10] and parabolic amplifier systems [parabolic], since gain-narrowing of the pulse bandwidth limits the minimum pulse duration. Existing experimental and numerical approaches for optimising cascaded amplifier chains soon become overly complex for all but the most experienced researchers. Recently we have used commercial telecommunications software developed for Erbium amplifiers by "VPI systems" with a customised module to allow for modeling of Yb amplifiers. Preserving the bandwidth of our femto-second modelocked oscillator [parabolic] in a system combining core- and cladding-pumped amplifiers was a key challenge. Each amplifier design was optimised to provide a combination of high-gain and broadbandwidth. The individual amplifier designs were then used in a cascaded simulation to predict the input pulse for the next amplifier stage[11]. Despite the complexities of working with a four stage amplifier system we achieved good agreement between experiment and theory.

High power pulsed fiber laser sources have been demonstrated over a range of many orders of magnitude in pulse duration and repetition rate, including sub-100fs systems and multi-GHz systems, operating at average powers up to several hundred watts. They offer the prospect of affordable, robust systems for a very wide variety of applications.

[1] N. G. R. Broderick, H. L. Offerhaus, D. J. Richardson, and R. A. Sammut, IEEE Photon. Technol. Lett. 10, 1718, (1998).

[2] K. Vysniauskas, L. M. B. Hickey, S. Alam, et. al., Photonics West 2005 San Jose

[3] F Ghiringhelli, K Vysniauskas, L M B Hickey, et. al. CLEO Europe 2005, CJ3-5-MON

[4] B.C.Thomsen, Y.Jeong, C.Codemard, et. al., CLEO/IQEC 2004 San Francisco CMAA

[5] P.Dupriez, A.Piper, A.Malinowski, OFC 2005 Anaheim 6-11 Mar 2005 (Postdeadline)

[6] P.Dupriez, C.Farrell, M.Ibsen, et. al., Photonics West 2006 San Jose, CA 21-16 Jan 2006

[7] P.Dupriez, J.K.Sahu, A.Malinowski, et. al., CLEO/QELS 2006 Long Beach, California 21-26 May 2006 CThJ1

[8] H.D.Foreman, K.G.Wilcox, A.C.Tropper, et. al., ASSP 2006 Nevada 29 Jan - 1 Feb 2006

[9] A. Malinowski, A. Piper, J. H. V. Price, et. al., Optics Letters, 29, 2073-2075, (2004)

[10] A. Galvanauskas, IEEE Journal of Selected Topics in Quantum Electronics, 7, 504-517, (2001)

[11] F. He, J.H.V. Price, D. J. Richardson, CLEO/QELS 2006 Long Beach, California 21-25 May 2006 